\title{
Child deaths: confidential enquiry into the role and quality of UK primary care
}

Anthony Harnden, Richard Mayon-White, David Mant, Deirdre Kelly and Gale Pearson

\begin{abstract}
Background

In 2006 the Confidential Enquiry into Maternal and Perinatal Deaths was extended to pilot a collection of child deaths. This helped optimise data collection for local safeguarding children's boards, which, since April 2008 , have a statutory responsibility to review all child deaths. Reviewing primary care records may highlight areas in which systems, skills, and care could be improved.
\end{abstract}

Aim

To review the role and quality of primary care in child deaths.

\section{Design of study}

Confidential enquiry into child deaths.

\section{Setting}

Five regions of the UK: North-East, South-West and West Midlands, Wales, and Northern Ireland.

\section{Method}

The confidential enquiry collected core data for all child deaths (age range 28 days to 17 years) and an agestratified sample was assessed by multidisciplinary panels for avoidable factors. An independent detailed review was conducted of the primary care records on all children in the North-East region and all children who were reviewed by panel in the other four regions.

Results

Primary care records were reviewed for 168 child deaths. There were $25(15 \%)$ deaths from acute infection, 22 (13\%) from cancer, and $11(7 \%)$ from asthma or epilepsy. Fifty-nine (35\%) deaths were sudden: sudden unexplained death in infancy, suicides, and assaults. Of 149 with immunisation records, only $88(59 \%)$ had been fully vaccinated on time. One or more primary care professionals were involved in the management of $90(54 \%)$ children. Avoidable primary care factors were identified in $18(20 \%)$ of these deaths. Avoidable primary care factors included failure in the recognition and management of serious infection, failure to vaccinate, and inadequate management of asthma and epilepsy.

\section{Conclusion}

Decisions made about diagnosis and management in primary care may affect the cause, time, and circumstances of a child's death. Maintaining skills in assessing the acutely ill child remains an essential part of good clinical practice.

Keywords

avoidable factors; child; death; primary health care.

\section{INTRODUCTION}

The standardised childhood death rate in the UK is 2.5 per $10000 .^{1}$ An average-sized practice with 10000 patients including 1500 children will have a child death about every 2 years. Because of their rarity in primary care and their impact on families, it is usual for a practice to reflect on the critical events that occurred in such instances and the involvement of members of the primary healthcare team. Indeed, child deaths are always remembered by the clinicians involved for many years afterwards. However, it is important that practices can learn the lessons from all childhood deaths, not just those of which they have direct experience. Critical event analysis can be a powerful learning tool when applied to a large number of deaths on a national scale. ${ }^{2}$

In 2006 the National Patient Safety Agency funded the Confidential Enquiry into Maternal and Child Health (CEMACH) to conduct a review of all childhood deaths in five regions of the UK. The aims were to identify avoidable factors and emerging healthcare themes and to inform future child death reviews including those of the recently established local safeguarding children boards. An independent study was undertaken within this review to identify themes and avoidable factors associated with primary health care.

A Harnden, FRCGP, FRCPCH, university lecturer in general practice; $\mathbf{R}$ Mayon-White, FRCP, consultant epidemiologist; D Mant, FRCGP, professor of general practice, Department of Primary Health Care, University of Oxford, Oxford. D Kelly, $M D, F R C P$, FRCPCH, chair, National Advisory Committee for the Child Health Enquiry; G Pearson, FRCPCH, clinical director, Child Health Enquiry, Confidential Enquiry in Maternal and Child Health, London.

Address for correspondence

Anthony Harnden, Department of Primary Health Care, University of Oxford, Old Road Campus, Headington, Oxford OX3 7LF. E-mail: anthony.harnden@dphpc.ox.ac.uk

Submitted: 19 March 2009; Editor's response: 15 April 2009; final acceptance: 8 May 2009

(c)British Journal of General Practice.

This article was originally online first on 2 Sep 2009. Cite this article as: Br J Gen Pract 2009; 59: 819-824. Advance online publication. DOI: 10.3399/bjgp09X472520 


\section{How this fits in}

National confidential enquiries can identify avoidable factors in maternal,

perinatal, and child deaths and make recommendations to improve clinical care. GPs have an important role in the care of children and are involved in providing clinical care for more than half of the children who die. This study utilising national data has identified themes and avoidable primary care factors in child deaths. Decisions made about diagnosis and management in primary care may affect the cause, time, and circumstances of a child's death.

\section{METHOD}

Data collected by the confidential enquiry enabled conduction of an independent primary care review. Complete primary care records were ascertained for 168 children (aged 28 days to 17 years) - all 92 children who died in the North-East region and an age-stratified sample of 76 children who died from four other geographical regions: England (South-West and West Midlands), Wales, and Northern Ireland. Of these 168 children, 87 were boys and 81 were girls. Thirty-six (21.4\%) were <1 year old, 30 (17.8\%) were aged 1-4 years, 30 (17.8\%) aged 5-9 years, 24 (14.3\%) aged 10-14 years, and 48 (28.6\%) aged 15-17 years. There were fewer infants and more teenagers in this primary care study than in the whole CEMACH review, in which 316 (33.0\%) children out of 957 were aged <1 year, and 209 (21.8\%) were teenagers aged $15-17$ years. $^{3}$

The core $\mathrm{CEMACH}$ dataset was limited to basic data about the number of children with primary care contact in the 3 months before death. While some panels discussed primary care involvement in cases that were selected for review, two of the researchers conducted an independent primary care-focused review of all available records for 168 children. The following were looked for: avoidable factors that were relevant to primary care; good and poor practice; and information that could be collected from primary care for future reviews of child deaths. The primary care records included the notes made by GPs, practice nurses, school nurses, and health visitors (where available), and the letters that GPs had received from hospitals and clinics. Most GP records had been computerised, with clear summaries of the events, medications, and correspondence. The other available records included hospital notes, correspondence, postmortem reports and, in some cases, newspaper cuttings. The study sought to identify and abstract any information from these other records that related to primary care and the cause of death.

In assessing whether anything might have been done in primary care to prevent the death, factors that a primary care practitioner might have been able to influence were sought. This does not imply proof that the death of an individual child should have been prevented, or even that the factor would have been removed if the primary care practitioner had taken appropriate action. However, if a factor was identified that contributed to a death which might have been ameliorated by action in primary care, it was classified as an avoidable primary care factor. In addition, the study identified possible avoidable factors that were of interest because there was potential to remove the factor by prevention delivered in primary care. An unavoidable death was one caused by factors wholly outside the influence of primary care, or caused by undetectable, unpreventable, and/or incurable disease.

The records were read independently and the researchers met to check the information abstracted and to identify and discuss issues that were common to a number of deaths. Specifically, the researchers looked at whether the GP was consulted for the fatal illness or injury, relevant preventive medical interventions, and features suggesting a risk of the fatal condition. It was noted whether the children had received their routine immunisations according to the national schedule, and the number of consultations for injuries in the children's lives were counted. The sequences of primary care consultations and treatments that were relevant to the cause of death were summarised. Illustrative case histories were sought, exemplifying themes that were common to a number of deaths. The personal details of illustrated cases have been changed to preserve confidentiality.

\section{RESULTS}

\section{Primary care involvement}

Of the 168 children for whom detailed primary care records were available, the extent of primary care involvement is shown in Table 1. In 90 deaths (54\%), one or more primary care professionals had seen the child within 3 months prior to death. In over two-thirds of cases $(64 / 90,71 \%)$, the child was receiving care for an existing illness, particularly congenital or neonatal conditions $(n=33)$ or cancer $(n=22)$. GPs were the primary care professionals involved in 76 of the cases, practice and community nurses in eight (with GPs in 7/8), a health visitor in one, and out-of-hours primary care services in five. In three cases, the GP's involvement was attempted resuscitation: a sudden unexplained death in infancy (SUDI), a child who choked on a plastic bag, and a teenager who collapsed with a sudden cardiac death during exercise. The commonest causes of death in cases without primary care involvement were SUDI and accidents.

\section{Diagnosis of acute life-threatening illness}

There were 25 deaths due to acute infections. In the 18 children with primary care involvement, recognition of 


\begin{tabular}{|c|c|c|c|}
\hline \multirow[b]{2}{*}{ Primary care actions } & \multicolumn{3}{|c|}{ Deaths, $n(\%)$} \\
\hline & $\begin{array}{l}\text { Primary care } \\
\text { involved }\end{array}$ & $\begin{array}{l}\text { Primary care } \\
\text { not involved }\end{array}$ & Total \\
\hline \multicolumn{4}{|l|}{ Diagnosis of and response to acute illness } \\
\hline Infection & $18(11)$ & $7(4)$ & $25(15)$ \\
\hline Other acute illness & $2(1)$ & $3(2)$ & $5(3)$ \\
\hline Management of asthma and epilepsy & $9(5)$ & $2(1)$ & $11(7)$ \\
\hline Diagnosis and palliative care for cancer & $22(13)$ & 0 & $22(13)$ \\
\hline Management of the effects of congenital, neonatal, and degenerative disabilities & s $33(20)$ & $13(8)$ & $46(27)$ \\
\hline Sudden deaths & $6(4)$ & $53(32)$ & $59(35)$ \\
\hline Total & $90(54)$ & $78(46)$ & $168(100)$ \\
\hline
\end{tabular}

the severity of the illness was the critical element (Box 1). Five of these deaths with primary care involvement were from bacterial meningitis and meningococcal septicaemia, four from pneumonia, two from invasive pneumococcal disease, and one each from bronchiolitis, croup, empyema, gastroenteritis, pericarditis, pertussis, and septicaemia secondary to chickenpox. The seven children who died of acute infection without primary care involvement had been taken directly to hospital (septicaemia in three, meningitis in two, empyema in one, and pneumonia in one). Two children died from other acute illnesses with primary care involvement (pulmonary embolism and a strangulated internal hernia). Two children died from brain haemorrhages, and one from intussusception, without primary care involvement

\section{Immunisation}

The primary care records were a good source of the immunisation histories for 149 of the 168 children in the study. Of the remainder, nine children were $<2$ months old (too young to start scheduled vaccines), and for 10 children the information was missing. Of the 149 children aged $\geq 2$ months with immunisation records,

\section{Box 1. Example of an avoidable primary care factor: failure to recognise severity of illness.}

A 12-year-old girl presented to her GP with illdefined symptoms of a flu-like illness lasting a week. She had coughed up blood on the day of presentation and her mother was alarmed. The GP recorded a temperature of $40.4^{\circ} \mathrm{C}$ and a clear chest on examination but thought she was hyperventilating and anxious. A diagnosis of a viral infection was made and the child was told to breathe into a paper bag to help the presumed hyperventilation. The GP wrote that a chest X-ray would be considered if the haemoptysis persisted. A date but not a time of consultation was recorded. She was taken to the hospital at 9.25 am the next day but died shortly afterwards of staphylococcal pneumonia.
88 (59\%) had been vaccinated according to the national schedule, 29 (19\%) had 1-2 months' delay in starting, 12 (8\%) had delays of 3-11 months in starting, and $8(5 \%)$ had longer delays in starting; 12 (8\%) had delays in completing the primary course, having started according to schedule. Of the 132 children who had reached their first birthday, 17 (13\%) had not completed a primary course of immunisation against diphtheria, tetanus and polio (DTP) and Haemophilus influenzae type B (Hib).

One child who had not started any primary immunisations died from pertussis aged 11 weeks. Both of the two children who died of invasive pneumococcal disease had congenital heart disease, an indication for conjugate pneumococcal vaccine. However, the GPs had not applied the recommendations of hospital specialists and contemporary national guidance (Box 2). The researchers considered failure to receive timely immunisations an avoidable primary care factor.

\section{Management of chronic illness}

Primary care was involved in the management of nine cases out of the 11 deaths from asthma and epilepsy. Three of the four asthma deaths were considered by the researchers to have avoidable primary care factors; the fourth occurred in a child with severe

\section{Box 2. Example of an avoidable primary care factor: failure to act on request for immunisation.}

A 9-month-old boy had congenital hypoplastic left heart syndrome which was repaired surgically. $\mathrm{He}$ had four respiratory infections, two managed by the GP at home and two that had resulted in hospital admissions. When he was aged 6 months, his hospital consultant wrote to the GP recommending pneumococcal vaccine. This advice was not followed. On the day that he died, he was 'chesty' but well enough to play in the morning; later he deteriorated rapidly with overwhelming pneumococcal septicaemia. 
Box 3. Example of lack of primary care involvement.

A 10-year-old boy with epilepsy was found dead at home. Apart from a home visit 10 months earlier for a respiratory infection, there were no recorded GP consultations over a 4-year period leading up to his death. Yet there were three hospital admissions via accident and emergency for seizures in his last 6 months of life. A hospital doctor had written to the parents advising on a change in the dose of anticonvulsant medication following an admission, and arranged for the child to be reviewed at a future outpatient appointment.

asthma treated according to current guidelines. One death from epilepsy had an avoidable primary care factor. In both diseases, there was evidence in the primary care records of missed appointments and uncoordinated care (Box 3). Three of the children with asthma and two of those with epilepsy had missed one or more appointments.

\section{Cancer}

GPs were involved to some degree in the care of all 22 children who died of cancer, from the initial diagnosis of cancer to palliative care during the last stage of illness. Between the diagnosis and terminal care, the primary care involvement was mainly limited to managing incidental problems and for routine visits such as vaccinations. While no primary care avoidable factors were identified, there were only a few examples of good palliative care delivered in the community.

\section{Support for children with congenital, neonatal, and degenerative diseases}

The largest group of deaths with GPs' and other primary care practitioners' involvement were 33 children with major congenital abnormalities and disabilities due to neonatal problems and degenerative neuromuscular disease. These children died from complications such as pneumonia and heart failure. The amount of involvement varied from close engagement with the problems as illustrated below, to being limited to prescribing treatments recommended by a hospital specialist. There were two children with congenital heart disease that was not diagnosed at the time of birth or at the first presentation to their GPs with illness.

Thirteen children who died from their disabilities had no primary care involvement. In four cases, this was because the children were very young and had remained under hospital care since birth. In the remaining seven cases the children's care had been hospital based, although primary care input might have been useful in four cases.

\section{Sudden deaths}

Primary care was directly involved in only six of the 59 sudden deaths (accidents, suicides, assaults, sudden cardiac death, and SUDI). In addition to the three resuscitation attempts, primary care practitioners were involved in the management of psychological problems in three children who committed suicide. The causes of 35 accidental deaths were road traffic accidents $(n=16)$, drowning $(n=6)$, falls $(n=4)$, drug overdoses $(n=3)$, fires $(n=2)$, choking $(n=2)$, a train accident, and a crush injury. For deaths from accidents, and those from assaults, it was difficult to determine any avoidable primary care factors since much of the responsibility for prevention (for example, road safety) lies with agencies unrelated to primary health care.

The primary care records of 156 children included reports from accident and emergency departments, as well as treatments given in primary care premises, thus enabling the researchers to count previous injuries. Twenty-nine children had one injury recorded and 24 had two or more, up to a maximum of eight. Fourteen children with more than one injury died in accidents. Eleven out 20 teenagers who died from accidental deaths had two or more previous injuries compared with four out of 37 teenagers who died from natural causes $(P<0.01)$.

\section{Avoidable factors}

Avoidable factors were identified in $18(20 \%)$ of the deaths for which primary care had involvement. This does not mean that these 18 deaths were preventable but that in each case a factor was identified that might have prevented death had it been corrected in primary care. Failure to recognise and manage severe infection was the most common avoidable primary care factor and occurred in 11 deaths. Examples of avoidable factors that might be ameliorated by action in primary care are given in Table 2.

For the whole group of 168 deaths, possible factors were identified in an additional $21(12 \%)$ deaths that might have been corrected with preventative advice delivered in primary care. For example, in the 15 SUDIs, the primary healthcare team had a role in advising on infant sleeping positions and feeding.

\section{DISCUSSION}

\section{Summary of main findings}

It was found that more than half of the children had been seen in primary care in the 3 months before death. Most of these encounters were with a GP. When GPs were involved in the immediate events leading to the child's death, there were avoidable primary care factors in $20 \%$.

While examples were found of outstanding care, (Box 4) in some cases poor decisions made in 
Table 2. Avoidable primary care factors.

\begin{tabular}{lll} 
Age & Primary care factor & Fatal consequence \\
\hline 1 month & Delay in hospital admission in neonate with respiratory distress & Pneumonia \\
\hline 2 months & Failure to recognise severity of respiratory infection & Pneumonia secondary to pertussis \\
\hline 4 months & Breathlessness attributed to bronchiolitis in child with congenital heart disease & Heart failure \\
\hline 7 months & Failure to administer pneumococcal vaccine on request & Pneumococcal meningitis \\
\hline 8 months & Failure to recognise severity of secondary infection in chickenpox & Septicaemia \\
\hline 9 months & Failure to administer pneumococcal vaccine on request & Pneumococcal septicaemia \\
\hline 1 year & Inadequate safety netting & Pneumococcal meningitis \\
\hline 1 year & Failure to recognise severe dehydration & E. coli diarrhoea \\
\hline 3 years & Cough and fever diagnosed as 'throat infection' & Empyema and septicaemia \\
\hline 6 years & Vomiting and pallor diagnosed as 'viral infection' & Viral pericarditis \\
\hline 8 years & Failure to attend follow-up GP appointment following asthma exacerbation & Severe acute asthma \\
\hline 8 years & Myalgia and fever diagnosed as 'flu' & Pneumococcal meningitis \\
\hline 8 years & Repeated failure to recognise severity of infection over 7 days & Viral pneumonia \\
\hline 12 years & Failure to recognise importance of respiratory symptoms & Staphylococcal pneumonia \\
\hline 14 years & Exceptionally reported from quality indicators for failure to attend & Acute asthma \\
\hline 15 years & Failure to follow up poor asthma control & Acute asthma \\
\hline 16 years & Prescribed epilepsy medication had run out & Drowned in bath during seizure \\
\hline 16 years & Severe acute infection diagnosed and treated as asthma & Pneumonia
\end{tabular}

primary care (Table 2) were the most important factors leading to the child's death.

\section{Strengths and limitations of the study}

A key strength of this study was that it was conducted using data from a well-established national confidential enquiry. As part of this process, the researchers gained access to high-quality primary care records and a rich source of additional data including hospital correspondence, post mortem reports, multi-agency records, and newspaper reports. The main limitation affecting this study is that a child's death is an exceptional event and the observations may not be uniformly applicable to all primary care. Interviews with parents and GPs were not part of the confidential enquiry process. An assessment of the timelines for acute illness would have been made easier by a recorded time of consultations in the primary care records. Future enquiries using case-control methodology could quantify the risk associated with primary care factors.

\section{Comparison with existing literature}

This is the first national study in the UK focusing on child deaths and primary care. A previous audit of 1263 deaths in four UK general practices found avoidable primary care factors such as delayed diagnosis, treatment, and referral in $5 \%$ of cases but few deaths were in children. ${ }^{4}$

In Arizona, US, a 5-year child fatality review found
2983 deaths attributable to medical conditions and $8 \%$ were judged to have been preventable. ${ }^{5}$ Inadequate paediatric emergency medical services, poor continuity of care, and delay in seeking medical care were described as preventable factors.

\section{Implications for clinical practice}

GPs should maintain their skills in the assessment of acutely ill children. Although precision of diagnosis for an evolving acute illness may not always be possible, parents should be empowered to reconsult should their child's condition deteriorate. A record of the time

\section{Box 4. Example of high-quality primary} care.

A 9-year-old girl who died suddenly at home had cystic fibrosis and spastic quadriplegia. She had GP care delivered from the same practice throughout her life. In the first year of life, the whole primary healthcare team offered support and advice about a range of issues including feeding, development, chest infections, diarrhoea, and seizures. Over the course of the girl's life, the primary care notes included clear records of communication to hospital specialists, a care planning agency, and a charitable grant-giving body for disabled children. The GP provided written fitness-to-travel certifications for the family on request. As well as good coordination of care, there was evidence that the child had regular reviews in the practice of medication, respiratory infections, diet, and development. 
of consultation and vital sign measurements ${ }^{6}$ may allow for a better assessment of the severity and rapidity of evolution of illness in any subsequent consultations. Serious illness such as childhood malignancy may also present over the time course of a few weeks in primary care.

Although initial management of childhood malignancy was usually hospital based, some GPs were proactive in contacting the family following the diagnosis. The knowledge that the GP is interested and caring may be all that is required immediately following diagnosis, and help to facilitate community care when it is required at a later stage of the illness. The care of children who are dying requires medical competence, teamwork, sensitivity, communication, and availability. In many cases, responsibility shared between hospital and primary care allowed a child's death to be managed very well in the community without the necessity for hospital admission. For the children who received no palliative care in the community, better provision of local support such as a community paediatric nurse might have allowed a child to die at home while still receiving continuity of care from their GP.

Premature infants may be at increased risk of infection, and in some cases delay in immunisation resulted in catastrophic consequences. At the time of this study conjugate pneumococcal vaccine was recommended for certain groups of at-risk children, although was not in the routine schedule. Yet there were unimmunised at-risk children who died from pneumococcal disease despite correspondence from the specialist to the GP recommending immunisation. One child died from varicella; immunisation for varicella is routine in some countries.

One recurrent theme to emerge among children who die was a failure to attend hospital appointments. Because it is not young children who default on appointments but their parents or carers, GPs should be aware that these children may be at increased risk of poorer health care. For this reason, practices could follow a consistent strategy of a member of the primary healthcare team proactively following up all such children.

Teenagers are a difficult group to reach out to because they access health care in an unstructured way. ${ }^{7}$ Although many vulnerable and at-risk teenagers in the present study had no contact with primary care, some had a history of contact with secondary care mental healthcare services. GPs should consider having a register of all children with mental health problems in primary care, and grasp the opportunity to offer preventative healthcare advice whenever a teenager is seen.

In April 2008 local safeguarding children boards were given a statutory responsibility for collecting and analysing information about every childhood death in England, with a view to identifying wider public health or safety concerns arising from a particular death. Primary care professionals should be involved in the review process, and primary care records should be part of the information they collect.

In this study, one-fifth of childhood deaths had avoidable factors related to primary care. The key lesson is anticipation and recognition that some children are at higher risk than others. Such children need more attention to vaccination against preventable infection and to management of underlying chronic illnesses. They need clinicians to adopt a lower threshold for investigation and referral when they present with new acute episodes of illness. The encouraging aspect of this study was to find examples of exceptionally high-quality primary care. The challenge is to ensure that the care of children is uniformly good across the whole of NHS primary care.

\section{Funding body}

The research was funded by a grant from the NIHR School for Primary Care Research entitled 'How can late diagnosis of serious infection in children be avoided?'

Ethic approval

North West Research Ethics Committee (reference 05/MRE08/51). The personal details of illustrated cases were changed to preserve confidentiality

\section{Competing interests}

The authors have stated that there are none

\section{Acknowledgements}

This work owes much to the help of the regional managers of $\mathrm{CEMACH}$, in particular Marjorie Renwick and her team in the Northern region, in collecting primary care records. We thank Richard Congdon, chief executive of CEMACH and Peter Rose, GP, for helpful comments on earlier drafts of the manuscript.

\section{Discuss this article}

Contribute and read comments about this article on the Discussion Forum: http://www.rcgp.org.uk/bjgp-discuss

\section{REFERENCES}

1. Office for National Statistics. Mortality statistics general: review of the Registrar General on deaths in England and Wales, 2004. Series DH1 no 37.

http://www.statistics.gov.uk/downloads/theme_health/Dh1_37_2004/ DH1_No_37.pdf (accessed 29 May 2009).

2. Lewis G (ed.). The Confidential Enquiry into Maternal and Child Health (CEMACH). Saving mothers' lives: reviewing maternal deaths to make motherhood safer 2003-2005. The seventh report on confidential enquiries into maternal deaths in the United Kingdom. London: CEMACH, 2007.

3. Pearson GA (ed.). Why children die: a pilot study 2006; England (South West, North East, West Midlands), Wales and Northern Ireland. London: CEMACH, 2008.

4. Holden J, O'Donnell S, Brindley J, Miles L. Analysis of 1263 deaths in four general practices. Br J Gen Pract 1998; 48(432): 1409-1412.

5. Rimsza ME, Schackner RA, Bowen KA, Marshall W. Can child deaths be prevented? The Arizona Child Fatality Review Program experience. Pediatrics 2002; 110(1 Pt 1): el1.

6. Thompson M, Mayon-White R, Harnden A, et al. Using vital signs to assess children with acute infections: a survey of current practice. $\mathrm{Br} J$ Gen Pract 2008; 58(549): 236-241.

7. Jacobson $\mathrm{L}$. The RCGP Adolescent Task Group reaches its teenage years. Br J Gen Pract 2007; 57(542): 749. 